\title{
Chemical analysis of a triple system of A-type stars
}

\author{
Y. Frémat, P. Lampens and H. Hensberge \\ Royal Observatory of Belgium, Ringlaan 3, B-1180 Brussel \\ e-mail: yves.fremat@oma.be
}

\begin{abstract}
Components of multiple systems generally originate from the same protostellar environment. Their similarities or differences in surface chemical composition therefore relates to their individual evolutionary paths (stellar evolution, rotation) and the possible influence of a close companion.

DG Leo is a spectroscopic hierarchical triple system with almost equal-mass late-A type components. Observations with high time and high spectral resolution were used to disentangle the spectrum of each component from the composite spectra. A detailed abundance analysis of the component spectra reveals that the wide companion has a nearly solar-like composition, while both components in the close binary show Am type peculiarities.
\end{abstract}

Keywords. Stars: abundances, stars: fundamental parameters, binaries: spectroscopic, stars: variable: $\delta$ Sct, stars: individual: (DG Leo).

\section{Introduction}

The analysis of the chemical composition of multiple systems having at least one pulsating component is an ideal tool to explore in an empirical way the interactions that may or may not exist between pulsation, diffusion, rotation and binarity. In the present study, we are, therefore, dealing with the chemical analysis of DG Leo (HD 85040, HR 3889, HIP 48218, Kui 44), a known multiple system with at least one pulsating component. DG Leo consists of a close binary (components $\mathrm{Aa}$ and $\mathrm{Ab}$ in a circular orbit) and one distant companion (component B). All three components were classified as late A-type stars. The orbital period of the Aa,b system is 4.15 days (Danziger \& Dickens 1967; Fekel \& Bopp 1977) while the orbital period of the visual pair Aab,B was estimated to be roughly 200 years (Fekel \& Bopp 1977). All three components of the system are potential candidates for pulsations as well as for Am peculiarities (they are located in the cooler part of the $\delta$ Scuti instability strip). In fact multiple short-period oscillations of type $\delta$ Scuti with periodicities of about 2 hrs have been detected (Lampens et al. 2005).

\section{Observations}

Extensive spectroscopy was obtained at the Haute-Provence Observatory (OHP) with the ELODIE spectrograph on the 1.93-m telescope. Observations were collected during 7 of the 8 allocated nights in 2003 (January $3-7 \&$ January $11-15$ ) and cover about $50 \%$ of the close binary's (components Aab) orbital phase. The time exposure was fixed at $360 \mathrm{~s}$ to resolve the presumed p-mode pulsations, yet with a good signal-to-noise ratio and a high resolution (about 50000). 245 spectra covering $\lambda \lambda 3900$ to 6800 were obtained with a signal-to-noise ratio that generally varies between 100 to 180 at $\lambda 5500$. 


\section{Spectral disentangling}

To extract the individual contribution of the three components, we adopted the spectral disentangling technique introduced by Hadrava (1995, see references therein) and applied in the KOREL computer code (Release 21.3.99). KOREL assumes that the observed spectrum is composed by $\mathrm{n}$ (three in our case) time-independent intrinsic spectra moving relatively to each other. It fits the Fourier Transform of the observations at different orbital phases by a Least-Squares Method to provide the time-averaged spectrum of each component (see Fig. 1) and their relative radial velocities.

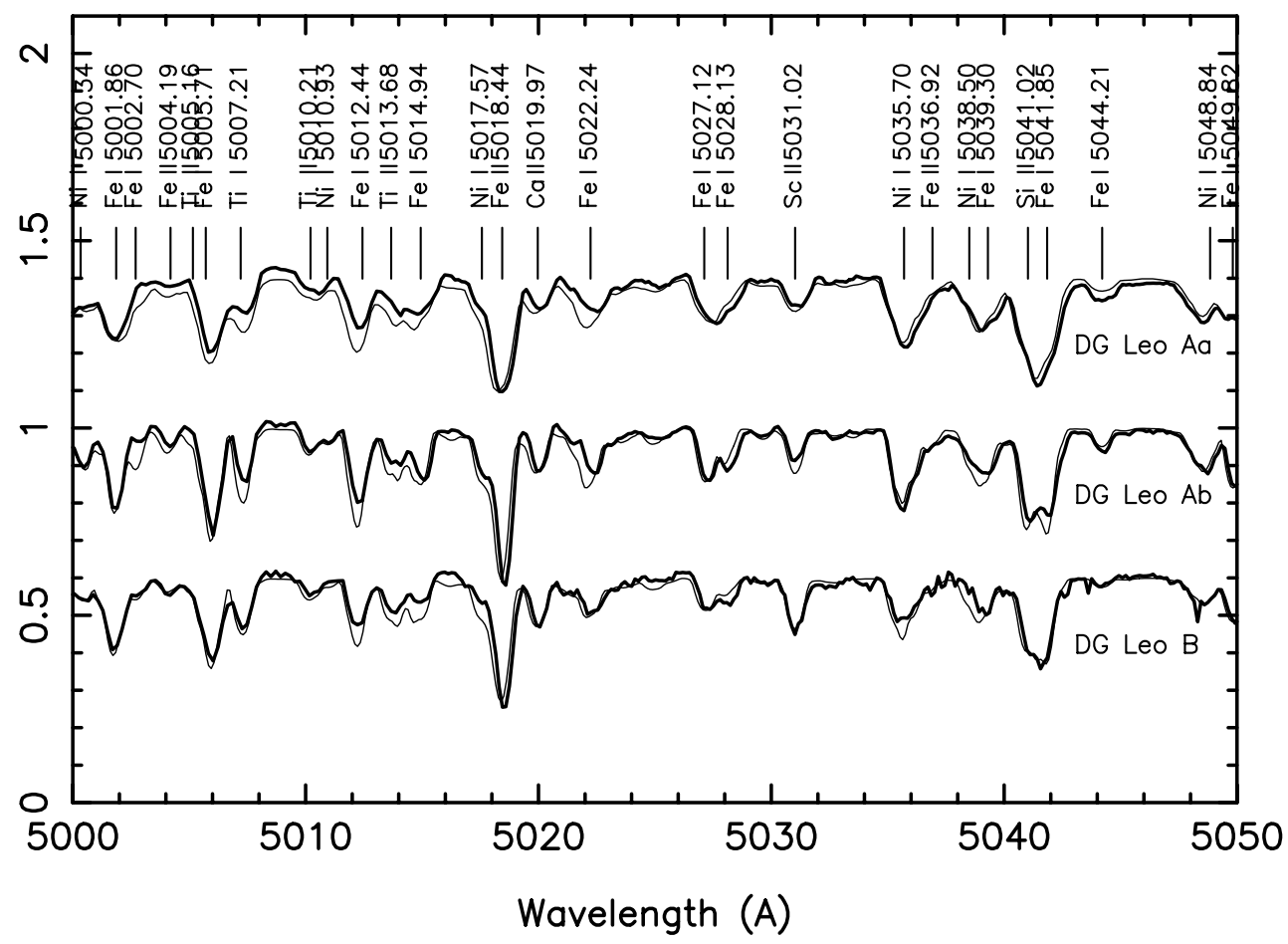

Figure 1. Component spectra. Comparison between the disentangled component spectra (thick line) and the synthetic spectra resulting from the chemical abundance analysis (thin line).

\section{Fundamental parameters}

The fundamental parameters that describe the components of DG Leo were derived, using the disentangled component spectra, in three consecutive steps. 1. The projected rotation velocity, $v \sin i$, was obtained performing a Fourier analysis of several unblended line profiles and using a procedure similar to the one intensively applied by Royer et al. (2002). 2. We estimated the effective temperature, $T_{\text {eff }}$, of DG Leo's components by fitting the individual $\mathrm{H} \alpha$ and $\mathrm{H} \gamma$ lines with theoretical line profiles computed in LTE using the SYNSPEC computer code (Hubeny \& Lanz 1995, and references therein) combined to ATlas9 (Kurucz 1993; Castelli et al. 1997) model atmospheres. 3. The surface gravity, $\log \mathrm{g}$, of the stars was determined from the HIPPARCOS parallax according to a method described by North et al. (1997). The luminosity was calculated using the parallax and the component's V magnitude while the stellar mass and radius were then interpolated in the theoretical evolutionary tracks $(Z=0.02)$ of Schaller et al. (1992). 


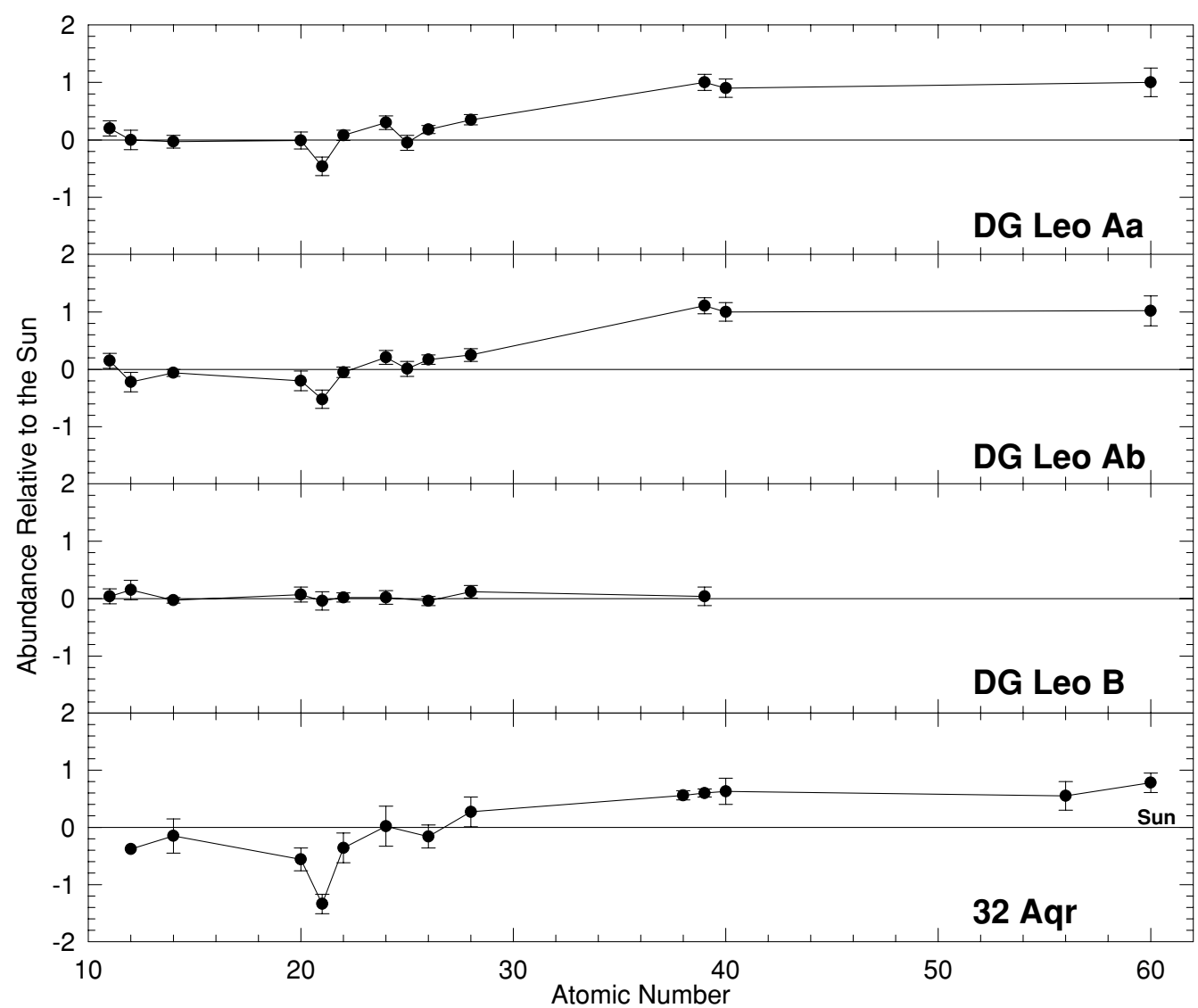

Figure 2. Chemical composition of DG Leo's components compared to the Sun and to the Am star 32 Aqr (Kocer et al. 1993). Component B has a solar-like chemical composition while both A components show abundance patterns typical of Am stars.

Table 1. Component fundamental parameters. $v \sin i$, and $\log g$ were derived using, respectively, the classical Fourier transform technique. Hydrogen line modelisation and theoretical evolution tracks. $\xi_{\text {turb }}$ is derived from the abundance analysis.

\begin{tabular}{lccc}
\hline component & Aa & Ab & B \\
\hline$T_{\text {eff }}(\mathrm{K})$ & $7470 \pm 220$ & $7390 \pm 220$ & $7590 \pm 220$ \\
$\log g$ & $3.8 \pm 0.14$ & $3.8 \pm 0.14$ & $3.8 \pm 0.12$ \\
$V \sin i\left(\mathrm{~km} \mathrm{~s}^{-1}\right)$ & $42 \pm 2$ & $28 \pm 2$ & $31 \pm 3$ \\
$\xi_{\text {turb }}\left(\mathrm{km} \mathrm{s}^{-1}\right)$ & $2.3 \pm 0.5$ & $2.3 \pm 0.5$ & $2.5 \pm 0.5$ \\
$M_{\text {HR }}(M \odot)$ & $2.0 \pm 0.2$ & $2.0 \pm 0.2$ & $2.1 \pm 0.2$ \\
\hline
\end{tabular}

\section{Chemical composition}

The atmospheric abundance analysis was then carried out by fitting theoretical line profiles to the observed component's spectra (see Fig. 1). Oscillator strengths, energy levels and damping parameters (including Stark, van der Waals and natural broadening) used during the fitting procedure were basically those compiled in the VALD-2 database (Kupka et al. 1999) updated by Erspamer \& North (2002). The final results are plotted in Fig. 2. 


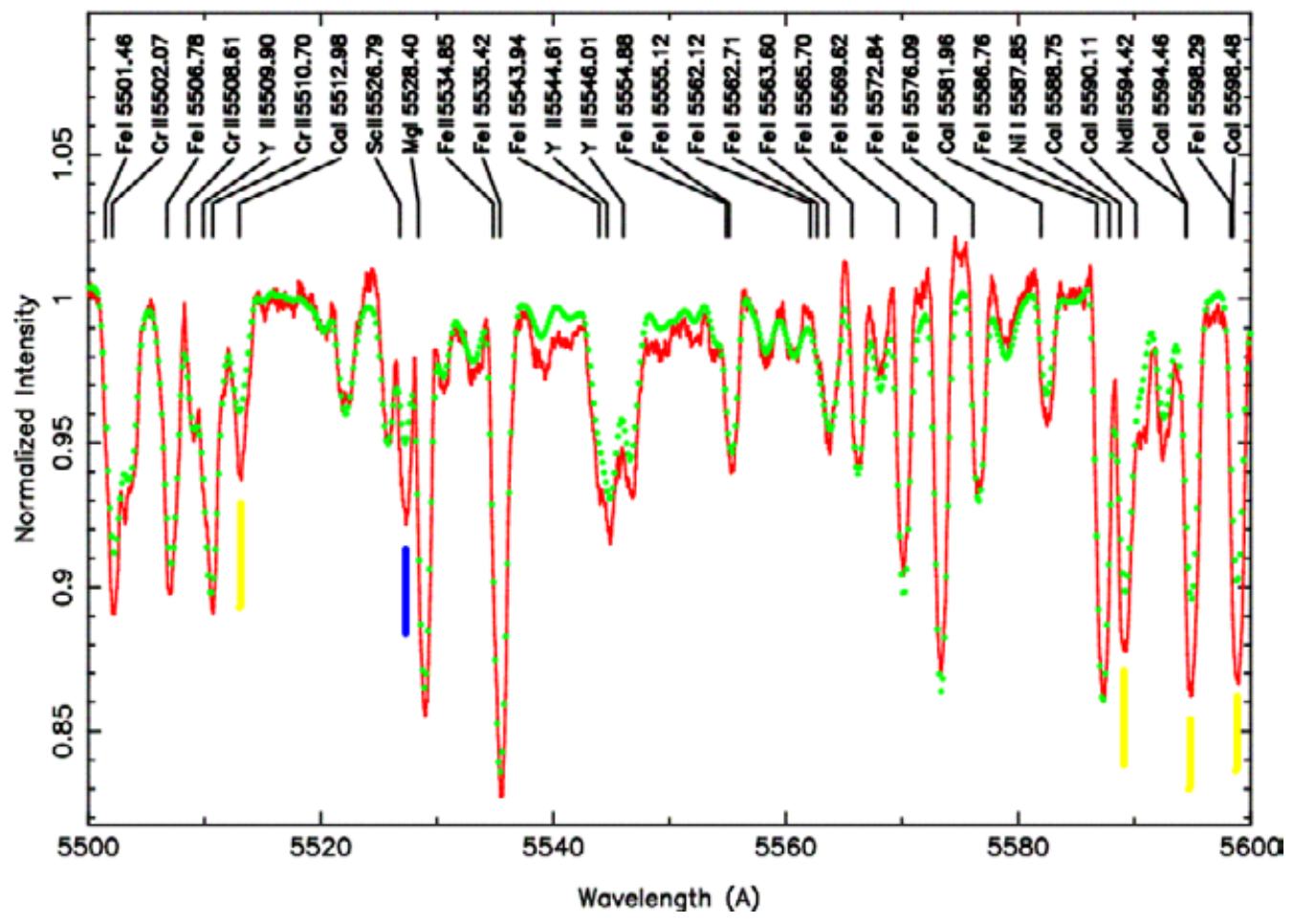

Figure 3. The spectra of DG Leo Aa (line) and DG Leo Ab (dots) are compared to each other. The spectrum of the latter component was convolved with a Gaussian function to artificially increase its projected rotation velocity. Some differences between the underabundances for scandium (blue line) and more strongly for calcium (yellow line) are obvious.

\section{Conclusions}

The Fourier Transform spectral disentangling technique (Hadrava 1995) was successfully applied to the study of the pulsating triple system DG Leo and a full description of this application and its results were submitted for publication (Frémat et al. 2004). Our conclusions follow: 1. All three stars have very similar fundamental parameters, 2. Component B has a solar-like chemical composition while Aa and Ab show Am-like over- and under-abundances, 3. Differences between the underabundances derived for scandium, and more strongly for calcium, are noted between the two A components (see Fig. 3), 4. though the close orbit is circularized, the rotation of at least one of the A components (which have different $\mathrm{v}$ sin i values) is apparently not synchronized with the orbital motion.

\section{Acknowledgements}

This work is based on observations made at the Haute-Provence Observatory (France). We thank G.Alecian for an interesting discussion about diffusion and we are grateful to P. Mathias, P. North \& E. Oblak for their help in gathering new radial velocity data. YF and PL acknowledge funding from the Belgian Federal Science Policy (Research project $\mathrm{MO} / 33 / 007$ ) and from the FNRS (Travel grant to Dubrovnik). HH acknowledges support from the IAP P5/36 project of the Belgian Federal Science Policy. 


\section{References}

Castelli, F., Gratton, R. G., \& Kurucz, R. L. 1997, A\& A, 318, 841

Danziger, I. J. \& Dickens, R. J. 1967, ApJ, 149, 55

Erspamer, D. \& North, P. 2002, A\&A A, 383, 227

Fekel, F. C. \& Bopp, B. W. 1977, PASP, 89, 216

Frémat, Y., Lampens, P., \& Hensberge, H. 2004, submitted to MNRAS

Hadrava, P. 1995, A\& AS, 114, 393

Hubeny, I. \& Lanz, T. 1995, ApJ, 439, 875

Kocer, D., Adelman, S. J., Bolcal, C., \& Hill, G. 1993, in ASP Conf. Ser. 44: IAU Colloq. 138: Peculiar versus Normal Phenomena in A-type and Related Stars, eds. M. M. Dworetsky, F. Castelli \& R. Faraggiana, 213

Kupka, F., Piskunov, N., Ryabchikova, T. A., Stempels, H. C., \& Weiss, W. W. 1999, A\&AS, 138,119

Kurucz, R. L. 1993, CD-ROM No.13. Cambridge, Mass.: Smithsonian Astrophysical Observatory.

Lampens, P., Frémat, Y., Garrido, R., et al. 2005, These Proceedings, GP18

North, P., Jaschek, C., \& Egret, D. 1997, in ESA SP-402: Hipparcos - Venice '97, 367

Royer, F., Gerbaldi, M., Faraggiana, R., \& Gómez, A. E. 2002, A\&A, 381, 105

Schaller, G., Schaerer, D., Meynet, G., \& Maeder, A. 1992, A\&AS, 96, 269 